\title{
New directions in knowledge-based personal computing
}

\author{
Jieun Lee ${ }^{1}$. Supratip Ghose ${ }^{2}$ • Jungsoo $\mathrm{Han}^{3}$
}

Published online: 18 August 2021

(C) The Author(s), under exclusive licence to Springer-Verlag London Ltd., part of Springer Nature 2021

Due to the progress made in information/convergence technology, a large amount of data is collected in diverse fields such as lifestyle, industry, and the environment. Computing technology that takes advantage to draw a potential significance from a large amount of data is actively progressing. Knowledge with a diverse significance drawn from such data is used to support human decision-making processes in these diverse fields. In addition, knowledge-based computing is used to explore hidden or latent potential information. In personal computing, a new knowledge-based direction is discovered in health management, treatment recommendations, behavioral pattern analysis, etc. For industry and the environment, new knowledgebased directions are being discovered in production technology and machines, weather prediction, traffic congestion prediction, accident risk assessment, etc. As described, studies that discover new directions from knowledge in such diverse fields are being conducted by the government, private companies, and in academic circles. In addition, it is possible to discover many diverse directions with the continuous change and progress of knowledge-aware computing.

This issue presents researchers as well as hands-on workers who share and discuss their research performance and solutions concerning progress in knowledge-based personal computing that uncovers new directions. These new directions in knowledge-based personal computing may add values that

Jungsoo Han

jungsoo.han.k@gmail.com

Jieun Lee

jieun.lee.0072@gmail.com

Supratip Ghose

sgresearch@gmail.com

1 Korea Convergence Society, 65, Buldang 21-ro, Seobuk-gu, Cheonan-si, Chungcheongnam-do, South Korea

2 Deptartment of Computer Science and Engineering, University of Information Technology \& Sciences, Dhaka, Bangladesh

3 Division of Computer Engineering, Baekseok University, Baekseokdaehak-ro, Dongnam-gu,

Cheonan-si, Chungcheongnam-do, South Korea would enhance the quality of life for humankind through cooperation and sharing those new ideas among research and development teams. The other side of these research traits amid the forefronts of the developments may open up debate on the conflicting issues in the in knowledge-based personal which is worth pondering upon.

The paper by Jin et al. [1] examines the influence of personal characteristics on knowledge discovery through social media. This study selected knowledge discovery behaviors as parameters based on self-efficacy. It verifies the mediating effects between independent variables and dependent variables from the perspective of knowledge discovery based on psychological factors. The paper by Sim et al. [2] developed a mobile support web service system using feedback from the autonomous knowledge information to generate biometric movement and user-space information. The information concerning physical change and spatio-temporal change is autonomously collected and accumulated through many kinds of sensors and mobile devices, with diverse services requested by users comprehensively and efficiently provided. The paper by Seshadri et al. [3] uses the single-tone Goertzel algorithm with the knowledge and sub-filter techniques in a field programmable gate array. The proposed method makes a comparison among transposed, direct, and contracted arrangements. It uses a contracted arrangement structure to ensure parallel processing and implements the Goertzel algorithm dividing the filter coefficient and data into odd and even numbers to enhance the calculation speed of digital signal processing (DSP).

The paper by Cho et al. [4] proposes knowledge-based tracking of multi-objects by using a machine learning model for video surveillance. The optic flow-based tracker is used to predict the location of an object in the next frame based on the location of that object in the frame. An algorithm combining optical flow and a CNN detects the location of an object through knowledge-based mining to accurately select the location of the tracked targets. The paper by Kim et al. [5] proposes a deep learning model that uses hierarchical structural features to classify informal knowledge. This study solves the problem of automatic classification of large-scale data. This model is used to classify and explore large-scale 
data for unstructured knowledge classification that consists of text and classes that have complex structural features, such as words, sentences, items, and documents. The paper by Lim et al. [6] proposes a method that integrates user preferences in order to provide individualized top- $\mathrm{N}$ recommendations with an item knowledge-based similarity model. This method combines similarity-based approaches with knowledge-based similarities to solve the cold-start problem and the data sparsity problem by using a mixture of matrix factorization and content-based filtering in e-commerce environments.

The paper by Kim and colleagues et al. [7] proposes fault prediction system using knowledge-based power monitoring in a smart factory. This is composed of a power measuring system, a data analysis server, a knowledge-based web server, and a smartphone application. The authors designed and implemented an IoT-based power monitoring and data collection system capable of detecting and monitoring abnormal power consumption in smart factories. The process analyzes real-time transactions and exploits them to provide predictions about the failure of equipment. The paper by $\mathrm{Li}$ et al. [8] analyzes information retrieval from behavioral construction using data mining. This analysis provides and supports decision-making on social network platforms that receive extensive attention. The authors configure an information retrieval behavior model, provide engines for data mining in a mobile social network, and use an empirical method to run tests. It is possible to play a mediating role in the process in which the relevant information has a user-conformity influence on information retrieval behavior. Park et al. [9] developed a lightweight, knowledge-based authentication model for intelligent CCTV video surveillance on a mobile personal computer. This model performs CCTV-RFID-enabled surveillance access control that can be used on a personally owned mobile device when it is difficult to perform accurate face recognition due to low-quality video or low-similarity feature vectors. In addition, their study introduces a lightweight user authentication protocol in knowledge and personal computing environments.

The paper by Jung et al. [10] proposes health lifelog mobile management using a knowledge-based blockchain network. They use a side-chain structure that saves information by applying various lifelog transactions and multi-context information to handle blockchain transactions, which are difficult to counterfeit or falsify on a knowledge-based health platform. The system saves continuously accumulated large-scale lifelog and multi-context information on the knowledgebased health block transactions. The paper by Nandhini et al. [11] proposes an algorithm that identifies errors and predicts optimal processes while utilizing multiple resources in cloud-based knowledge computing. This identifies and predicts different levels of errors and takes into consideration diverse types of resources to minimize error occurrences and process-execution time. It uses deadline-determined resource allocation with virtual machine resource isolation to overcome enhanced fault tolerance in optimal tasks. The paper by Park et al. [12] developed a cloud and long-range wide area network (LoRaWAN) communication-based container-type maritime knowledge system which is designed for monitoring maritime environment improvements. It uses an energyefficient method to collect and visualize long-range maritime environment data through the LoRaWAN communication module and analyzes convergence-type maritime devices through processing red tide prediction-related knowledge.

The paper by Chung et al. [13] proposes a dynamic knowledge-based multi-mode classification model. It is configured with a human learning principle-based CNN to classify multi-modal knowledge implemented via a multi-modal classification graphical user interface using deep learning. It provides feedback on the classification results, adjusting dynamic knowledge through generating convergence influence between diverse domain knowledge bases and multi-modal deep learning. The paper by Park et al. [14] analyzes non-group tourist experience enhancement factors to develop personalized smartphone-based services through big data information retrieval. This method uses influence factors from the positive effects of travel information acquisition and travel intentions by moderating independent and dependent variables. It predicts roles for non-group tourists based on the previous literature reviews of travel plan designs. The paper by Liu et al. [15] suggests a topic model that uses LDA and Word2Vec to recommend travel routes by focusing on travel reviews. The proposed method collects reviews prepared by tourists which not only performs network analysis, but also supports big data mining and text mining. It proposes a process for data collection, data cleaning, and data analysis in order to develop travel sites and travel applications using the result form the review analysis of text mining techniques.

The paper by Beak et al. [16] suggests a CNN-based classification model using influential health factors and knowledge mining. This study extracts influence factors required for health predictions through multivariate analysis, and analyzes the causal relationships between chronic diseases as well as their correlations. The analysis later identifies the factors diabetes, obesity, and high/low blood pressure. In addition, this study derives a knowledge health mining model from the associations among various influential factors. The paper by Xu et al. [17] suggests a knowledge-based edge computing framework with a network that enables heterogeneous connections using IoT devices under CoAP and HTTP protocols. This framework includes the overall implementation and distribution range of edge computing using the EdgeX platform for the IoT and uses dispersed micro-service application technology to provide better maintenance and expandability. This paper presents EdgeX connected to heterogeneous devices, and then, the CoAP and HTTP protocol-based REST API is implemented in an intelligent micro-service. 
The paper by Lee et al. [18] proposes a deep learningbased health model using knowledge mining to predict heart disease cases throughout the 1-year follow-up period after a patient with acute myocardial infarction is discharged from hospital. This model uses knowledge mining to predict abnormal reactions that patients with acute myocardial infarction show throughout their 1-, 6-, and 12-month post-hospitalization follow-up periods. The paper by Hong et al. [19] develops a knowledge-based mobile multimedia system using candidate features and accident frequency to predict traffic accident risks. This study uses a multilayer perceptron model to estimate from time series analysis the risk level for traffic accidents. It entails knowledge on the emerging levels of risk for traffic accidents depending on various situations. In addition, it is possible to provide road safety guidance services and mobile quick route guidance services. The paper by Chaurasia et al. [20] proposes a mobile-based technology adoption modeling process for patients with dementia. This study analyzes and merges the data that provide the background information, psychosocial information, and medical history information of the subjects. In addition, the optimal classification model is identified through feature selection and dimensionality reduction. This identifies the optimal data subset capable of providing better decision-making from the earlier existing adoption modeling.

This fine collection of articles was achieved by fruitful collaborations. We would like to thank Prof. Peter Thomas, the Editor-in-Chief of Personal and Ubiquitous Computing, for his great efforts and support throughout the publication.

\section{References}

1. Li G, Park EM, Jin SJ (2021) The impacts of personal traits on knowledge discovery behaviors via mobile SNS. Pers Ubiquit Comput. https://doi.org/10.1007/s00779-019-01240-1

2. Sim S, Cho M (2021) A study on Web service supporting mobility of users using ICT-based autonomous feedback knowledge. Pers Ubiquit Comput. https://doi.org/10.1007/s00779-019-01251-y

3. Seshadri R, Ramakrishnan S, Satheesh Kumar J (2021) Knowledge-based single-tone digital filter implementation for DSP systems. Pers Ubiquit Comput. https://doi.org/10.1007/ s00779-019-01304-2

4. Ahn H, Cho HJ (2021) Research of multi object detection and tracking using machine learning based on knowledge for video surveillance system. Pers Ubiquit Comput. https://doi.org/10. 1007/s00779-019-01296-Z

5. Joo W, Choi K, Kim YK (2021) Deep learning model for unstructured knowledge classification using structural features. Pers Ubiquit Comput. https://doi.org/10.1007/s00779-019-01244-x
6. Yang Y, Jo J, Lim H (2021) Unifying user preference and item knowledge based similarity models for top-N recommendation. Pers Ubiquit Comput. https://doi.org/10.1007/s00779-019-01252-x

7. Kim E, Huh DH, Kim S (2021) Knowledge-based power monitoring and fault prediction for smart factories. Pers Ubiquit Comput. https://doi.org/10.1007/s00779-019-01348-4

8. Jin H, Miao Y, Jung JR, Li D (2021) Construction of information search behavior based on data mining. Pers Ubiquit Comput. https://doi.org/10.1007/s00779-019-01239-8

9. Kim J, Park N (2021) Lightweight knowledge-based authentication model for intelligent closed circuit television in mobile personal computing. Pers Ubiquit Comput. https://doi.org/10.1007/s00779019-01299-w

10. Chung K, Jung H (2021) Knowledge-based block chain networks for health $\log$ data management mobile service. Pers Ubiquit Comput. https://doi.org/10.1007/s00779-019-01326-w

11. Nandhini JM, Gnanasekaran T (2021) Enhanced fault identification and optimal task prediction (EFIOTP) algorithm during multiresource utilization in cloud-based knowledge and personal computing. Pers Ubiquit Comput. https://doi.org/10.1007/s00779-01901265-6

12. Park S, Ling TC, Cha B, Kim J (2021) Correction to: Design of containerized marine knowledge system based on IoT-Cloud and LoRaWAN. Pers Ubiquit Comput. https://doi.org/10.1007/s00779020-01400-8

13. Park SS, Chung K (2021) MMCNet: deep learning-based multimodal classification model using dynamic knowledge. Pers Ubiquit Comput. https://doi.org/10.1007/s00779-019-01261-w

14. Li G, Seo JH, Park EM (2021) Factors enhancing independent tourists' experience through convergence of smartphone-based services and information searching. Pers Ubiquit Comput. https://doi. org/10.1007/s00779-020-01473-5

15. Park ST, Liu C (2021) A study on topic models using LDA and Word2Vec in travel route recommendation: focus on convergence travel and tours reviews. Pers Ubiquit Comput. https://doi.org/10. 1007/s00779-020-01476-2

16. Beak JW, Chung K (2021) CNN-based health model using knowledge mining of influencing factors. Pers Ubiquit Comput. https:// doi.org/10.1007/s00779-019-01300-6

17. Xu R, Jin W, Kim DH (2021) Knowledge-based edge computing framework based on CoAP and HTTP for enabling heterogeneous connectivity. Pers Ubiquit Comput. https://doi.org/10.1007/ s00779-020-01466-4

18. Kim YJ, Saqlian M, Lee JY (2021) Deep learning-based prediction model of occurrences of major adverse cardiac events during 1-year follow-up after hospital discharge in patients with AMI using knowledge mining. Pers Ubiquit Comput. https://doi.org/10.1007/ s00779-019-01248-7

19. Park RC, Hong EJ (2021) Urban traffic accident risk prediction for knowledge-based mobile multimedia service. Pers Ubiquit Comput. https://doi.org/10.1007/s00779-020-01442-y

20. Chaurasia P, McClean S, Nugent CD, Cleland I, Zhang S, Donnelly MP, Scotney BW, Sanders C, Smith K, Norton MC, Tschanz J (2021) Modelling mobile-based technology adoption among people with dementia. Pers Ubiquit Comput. https://doi.org/10.1007/ s00779-021-01572-x

Publisher's note Springer Nature remains neutral with regard to jurisdictional claims in published maps and institutional affiliations. 